\title{
Some new hybrid power mean formulae of trigonometric sums
}

Li Chen $^{1}$ and Zhuoyu Chen ${ }^{1 *}$

\author{
"Correspondence: \\ chenzymath@163.com \\ 'School of Mathematics, Northwest \\ University, Xi'an, P.R. China
}

\begin{abstract}
We apply the analytic method and the properties of the classical Gauss sums to study the computational problem of a certain hybrid power mean of the trigonometric sums and to prove several new mean value formulae for them. At the same time, we also obtain a new recurrence formula involving the Gauss sums and two-term exponential sums.

MSC: 11L05; 11L07

Keywords: Cubic Gauss sums; Two-term exponential sums; Hybrid power mean; Computational formula
\end{abstract}

\section{Introduction}

For any integer $m$ and odd prime $p \geq 3$, the cubic Gauss sums $A(m, p)=A(m)$ are defined as follows:

$$
A(m)=\sum_{a=0}^{p-1} e\left(\frac{m a^{3}}{p}\right)
$$

where, as usual, $e(y)=e^{2 \pi i y}$.

We found that several scholars studied the hybrid mean value problems of various trigonometric sums and obtained many interesting results. For example, Chen and $\mathrm{Hu}$ [1] studied the computational problem of the hybrid power mean

$$
S_{k}(p)=\sum_{m=1}^{p-1}\left(\sum_{a=0}^{p-1} e\left(\frac{m a^{3}}{p}\right)\right)^{k} \cdot\left|\sum_{c=1}^{p-1} e\left(\frac{m c+\bar{c}}{p}\right)\right|^{2},
$$

where $\bar{c}$ denotes the multiplicative inverse of $c \bmod p$, that is, $c \cdot \bar{c} \equiv 1 \bmod p$.

For $p \equiv 1 \bmod 3$, they proved an interesting third-order linear recurrence formula for $S_{k}(p)$.

(c) The Author(s) 2020. This article is licensed under a Creative Commons Attribution 4.0 International License, which permits use, sharing, adaptation, distribution and reproduction in any medium or format, as long as you give appropriate credit to the original author(s) and the source, provide a link to the Creative Commons licence, and indicate if changes were made. The images or other third party material in this article are included in the article's Creative Commons licence, unless indicated otherwise in a credit line to the material. If material is not included in the article's Creative Commons licence and your intended use is not permitted by statutory regulation or exceeds the permitted use, you will need to obtain permission directly from the copyright holder. To view a copy of this licence, visit http://creativecommons.org/licenses/by/4.0/. 
$\mathrm{Li}$ and $\mathrm{Hu}$ [2] studied the computational problem of the hybrid power mean

$$
\sum_{b=1}^{p-1}\left|\sum_{a=0}^{p-1} e\left(\frac{b a^{4}}{p}\right)\right|^{2} \cdot\left|\sum_{c=1}^{p-1} e\left(\frac{b c+\bar{c}}{p}\right)\right|^{2}
$$

and proved an exact computational formula for (1).

Zhang and Zhang [3] proved the identity

$$
\sum_{m=1}^{p-1}\left|\sum_{a=0}^{p-1} e\left(\frac{m a^{3}+n a}{p}\right)\right|^{4}= \begin{cases}2 p^{3}-p^{2} & \text { if } 3 \nmid p-1, \\ 2 p^{3}-7 p^{2} & \text { if } 3 \mid p-1 .\end{cases}
$$

Other related contents can also be found in [4-12], which will not be repeated here. In this paper, inspired by [1] and [2], we consider the following mean value:

$$
H_{k}(c, p)=\sum_{m=1}^{p-1}\left(\sum_{a=0}^{p-1} e\left(\frac{c m a^{3}}{p}\right)\right)^{k} \cdot\left(\sum_{a=0}^{p-1} e\left(\frac{m a^{3}+a}{p}\right)\right)^{3} .
$$

We do not know whether there exists a precise computational formula for (2), where $c$ is any integer with $(c, p)=1$, and $p \equiv 1 \bmod 3$.

Actually, there also exists a third-order linear recurrence formula of $H_{k}(c, p)$ for all integers $k \geq 1$ and $c$. But for some integers $c$, the initial value of $H_{k}(c, p)$ is very simple, whereas for other $c$, the initial value of $H_{k}(c, p)$ is more complex. So a satisfactory recursive formula for $H_{k}(c, p)$ is not available.

The main purpose of this paper is using an analytic method and the properties of classical Gauss sums to give an effective calculation method for $H_{k}(c, p)$ with some special integers $c$. We will prove the following two theorems.

Theorem 1 Let $p$ be a prime with $p \equiv 1 \bmod 3$. If 3 is not a cubic residue $\bmod p$, then we have

$$
\begin{aligned}
& \sum_{m=1}^{p-1}\left(\sum_{a=0}^{p-1} e\left(\frac{3 m a^{3}}{p}\right)\right)\left(\sum_{b=0}^{p-1} e\left(\frac{m b^{3}+b}{p}\right)\right)^{3}=3 p^{2}+d p^{2}, \\
& \sum_{m=1}^{p-1}\left(\sum_{a=0}^{p-1} e\left(\frac{m a^{3}}{p}\right)\right)^{2}\left(\sum_{b=0}^{p-1} e\left(\frac{m b^{3}+b}{p}\right)\right)^{3}=p^{2}(3 p-5 d),
\end{aligned}
$$

and

$$
\sum_{m=1}^{p-1}\left(\sum_{a=0}^{p-1} e\left(\frac{3 m a^{3}}{p}\right)\right)^{3}\left(\sum_{b=0}^{p-1} e\left(\frac{m b^{3}+b}{p}\right)\right)^{3}=p^{2}\left(5 d p+9 p-d^{2}\right) .
$$

Theorem 2 Let $p$ be an odd prime with $p \equiv 1 \bmod 3$. If 3 is a cubic residue mod $p$, then for any integer $k \geq 3$, we have the third-order linear recurrence formula

$$
H_{k}(1, p)=3 p H_{k-2}(1, p)+d p H_{k-3}(1, p)
$$

where the first three terms are $H_{0}(1, p)=2 p^{2}-p d, H_{1}(1, p)=p^{2}(d-6)$, and $H_{2}(1, p)=$ $p^{2}(6 p-5 d)$. 
Some notes: First, in Theorem 1, if $(3, p-1)=1$, then the question we are discussing is trivial, because in this case, we have

$$
\sum_{a=0}^{p-1} e\left(\frac{m a^{3}}{p}\right)=\sum_{a=0}^{p-1} e\left(\frac{m a}{p}\right)=0
$$

Second, in the first and third formulas of Theorem 1, we take $c=3$ (and $c=1$ in the second formula). These are all for getting the exact value of the mean value. Otherwise, the results will not be pretty.

\section{Several lemmas}

To complete the proofs of our theorems, several lemmas are essential. Hereafter, we will use related properties of the classical Gauss sums and the third-order character mod $p$, all of which can be found in books concerning elementary number theory or analytic number theory, such as [13] and [14]. First we have the following:

Lemma 1 Let $p$ be a prime with $p \equiv 1 \bmod 3$. Then for any third-order character $\psi \bmod p$, we have the identity

$$
\sum_{m=1}^{p-1} \psi(m)\left(\sum_{a=0}^{p-1} e\left(\frac{m a^{3}+a}{p}\right)\right)^{3}=\bar{\psi}(3) p \tau^{2}(\bar{\psi})-3 p \tau(\psi)
$$

Proof First, applying the trigonometric identity

$$
\sum_{m=1}^{q} e\left(\frac{n m}{q}\right)= \begin{cases}q & \text { if } q \mid n \\ 0 & \text { if } q \nmid n\end{cases}
$$

and noting that $\psi^{3}=\chi_{0}$, the principal character $\bmod p$, we have

$$
\begin{aligned}
\sum_{m=1}^{p-1} \psi(m) & \left(\sum_{a=0}^{p-1} e\left(\frac{m a^{3}+a}{p}\right)\right)^{3} \\
= & \sum_{m=1}^{p-1} \psi(m)\left(\sum_{a=0}^{p-1} e\left(\frac{m a^{3}+a}{p}\right)\right)^{2} \\
& +\sum_{m=1}^{p-1} \psi(m)\left(\sum_{a=0}^{p-1} e\left(\frac{m a^{3}+a}{p}\right)\right)^{2}\left(\sum_{a=1}^{p-1} e\left(\frac{m a^{3}+a}{p}\right)\right) \\
= & 2 \sum_{m=1}^{p-1} \psi(m) \sum_{a=1}^{p-1} e\left(\frac{m a^{3}+a}{p}\right)+\sum_{m=1}^{p-1} \psi(m)\left(\sum_{a=1}^{p-1} e\left(\frac{m a^{3}+a}{p}\right)\right)^{2} \\
& +\tau(\psi) \sum_{a=0}^{p-1} \sum_{b=0}^{p-1} \sum_{c=1}^{p-1} \bar{\psi}\left(a^{3}+b^{3}+c^{3}\right) e\left(\frac{a+b+c}{p}\right) \\
= & -2 \tau(\psi)+\tau(\psi) \sum_{a=1}^{p-1} \bar{\psi}\left(a^{3}+1\right) \sum_{b=1}^{p-1} e\left(\frac{b(a+1)}{p}\right)
\end{aligned}
$$




$$
\begin{aligned}
& +\tau(\psi) \sum_{a=0}^{p-1} \sum_{b=0}^{p-1} \bar{\psi}\left(a^{3}+b^{3}+1\right) \sum_{c=1}^{p-1} e\left(\frac{c(a+b+1)}{p}\right) \\
= & -2 \tau(\psi)-\tau(\psi) \sum_{a=1}^{p-1} \bar{\psi}\left(a^{3}+1\right)+p \tau(\psi) \sum_{\substack{a=0 \\
a+b+1 \equiv=0 \bmod p}}^{p-1} \sum_{b=0}^{p-1} \bar{\psi}\left(a^{3}+b^{3}+1\right) \\
& -\tau(\psi) \sum_{a=0}^{p-1} \sum_{b=0}^{p-1} \bar{\psi}\left(a^{3}+b^{3}+1\right) \\
= & -2 \tau(\psi)-\tau(\psi) \sum_{a=1}^{p-1} \bar{\psi}\left(a^{3}+1\right)+p \tau(\psi) \sum_{a=0}^{p-1} \bar{\psi}\left(a^{3}-(a+1)^{3}+1\right) \\
& -\tau(\psi) \sum_{a=0}^{p-1} \sum_{b=0}^{p-1} \bar{\psi}\left(a^{3}+b^{3}+1\right) .
\end{aligned}
$$

Noting that $\psi^{2}=\bar{\psi}$ and $\tau(\psi) \tau(\bar{\psi})=p$, from the properties of Gauss sums we have

$$
\begin{aligned}
\sum_{a=1}^{p-1} \bar{\psi}\left(a^{3}+1\right) & =\sum_{a=1}^{p-1} \bar{\psi}(a+1)(1+\psi(a)+\bar{\psi}(a)) \\
& =\sum_{a=1}^{p-1} \bar{\psi}(a+1)+\sum_{a=1}^{p-1} \bar{\psi}(1+\bar{a})+\sum_{a=1}^{p-1} \bar{\psi}\left(a^{2}+a\right) \\
& =-2+\frac{1}{\tau(\psi)} \sum_{b=1}^{p-1} \psi(b) \sum_{a=1}^{p-1} \bar{\psi}(a) e\left(\frac{b(a+1)}{p}\right) \\
& =-2+\frac{\tau^{2}(\bar{\psi})}{\tau(\psi)}=-2+\frac{\tau^{3}(\bar{\psi})}{p}, \\
\sum_{a=0}^{p-1} \bar{\psi}\left(a^{3}-(a+1)^{3}+1\right)=\sum_{a=0}^{p-1} \bar{\psi}(-3 a(a+1)) & \bar{\psi}(3) \sum_{a=1}^{p-1} \bar{\psi}(a(a+1))=\frac{\bar{\psi}(3) \tau^{3}(\bar{\psi})}{p} .
\end{aligned}
$$

Since $\psi$ is a third-order character $\bmod p$, for any integer $c$ with $(c, p)=1$, from the properties of the classical Gauss sums we have

$$
\sum_{a=0}^{p-1} e\left(\frac{c a^{3}}{p}\right)=1+\sum_{a=1}^{p-1}(1+\psi(a)+\bar{\psi}(a)) e\left(\frac{c a}{p}\right)=\bar{\psi}(c) \tau(\psi)+\psi(c) \tau(\bar{\psi}) .
$$

Applying (7), we have

$$
\begin{aligned}
\sum_{a=0}^{p-1} \sum_{b=0}^{p-1} \bar{\psi}\left(a^{3}+b^{3}+1\right) & =\frac{1}{\tau(\psi)} \sum_{c=1}^{p-1} \psi(c) \sum_{a=0}^{p-1} \sum_{b=0}^{p-1} e\left(\frac{c a^{3}+c b^{3}+c}{p}\right) \\
& =\frac{1}{\tau(\psi)} \sum_{c=1}^{p-1} \psi(c) e\left(\frac{c}{p}\right)\left(\sum_{a=0}^{p-1} e\left(\frac{c a^{3}}{p}\right)\right)^{2}
\end{aligned}
$$




$$
\begin{aligned}
& =\frac{1}{\tau(\psi)} \sum_{c=1}^{p-1} \psi(c) e\left(\frac{c}{p}\right)\left(\psi(c) \tau^{2}(\psi)+2 p+\bar{\psi}(c) \tau^{2}(\bar{\psi})\right) \\
& =\tau(\psi) \tau(\bar{\psi})+2 p-\frac{\tau^{3}(\bar{\psi})}{p}=3 p-\frac{\tau^{3}(\bar{\psi})}{p}
\end{aligned}
$$

Combining (4), (5), (6), and (8), we have the identity

$$
\sum_{m=1}^{p-1} \psi(m)\left(\sum_{a=0}^{p-1} e\left(\frac{m a^{3}+a}{p}\right)\right)^{3}=\bar{\psi}(3) p \tau^{2}(\bar{\psi})-3 p \tau(\psi)
$$

This proves Lemma 1.

Lemma 2 Let $p$ be a prime with $p \equiv 1 \bmod 3$, and let $\psi$ be any third-order character $\bmod p$. Then we have

$$
\tau^{3}(\psi)+\tau^{3}(\bar{\psi})=d p
$$

where $\tau(\psi)$ denotes the classical Gauss sums, and $d$ is uniquely determined by $4 p=d^{2}+$ $27 b^{2}$ and $d \equiv 1 \bmod 3$.

Proof See [4] or [9].

Lemma 3 Let $p$ be a prime with $p \equiv 1 \bmod 3$. Then we have the identity

$$
\sum_{m=1}^{p-1}\left(\sum_{a=0}^{p-1} e\left(\frac{m a^{3}+a}{p}\right)\right)^{3}=2 p^{2}-p d
$$

Proof Since the congruence equation $x^{3}+1 \equiv 0 \bmod p$ has three solutions in a reduced residue system $\bmod p$, from (3) we have

$$
\begin{aligned}
\sum_{m=1}^{p-1} & \left(\sum_{a=0}^{p-1} e\left(\frac{m a^{3}+a}{p}\right)\right)^{3} \\
= & \sum_{m=0}^{p-1}\left(\sum_{a=0}^{p-1} e\left(\frac{m a^{3}+a}{p}\right)\right)^{3} \\
= & \sum_{m=0}^{p-1}\left(\sum_{a=0}^{p-1} e\left(\frac{m a^{3}+a}{p}\right)\right)^{2}+\sum_{m=0}^{p-1}\left(\sum_{a=0}^{p-1} e\left(\frac{m a^{3}+a}{p}\right)\right)^{2}\left(\sum_{a=1}^{p-1} e\left(\frac{m a^{3}+a}{p}\right)\right) \\
= & p+2 \sum_{m=0}^{p-1} \sum_{a=1}^{p-1} e\left(\frac{m a^{3}+a}{p}\right)+\sum_{m=0}^{p-1}\left(\sum_{a=1}^{p-1} e\left(\frac{m a^{3}+a}{p}\right)\right)^{2} \\
& +\sum_{a=0}^{p-1} \sum_{b=0}^{p-1} \sum_{c=1}^{p-1} \sum_{m=0}^{p-1} e\left(\frac{m c^{3}\left(a^{3}+b^{3}+1\right)+c(a+b+1)}{p}\right) \\
= & p+\sum_{a=1}^{p-1} \sum_{b=1}^{p-1} \sum_{m=0}^{p-1} e\left(\frac{m b^{3}\left(a^{3}+1\right)+b(a+1)}{p}\right)
\end{aligned}
$$




$$
\begin{aligned}
& +p \sum_{\substack{a=0 \\
a^{3}+b^{3}+1 \equiv 0 \bmod p}}^{p-1} \sum_{c=1}^{p-1} e\left(\frac{c(a+b+1)}{p}\right) \\
& =p+p(p-1)-2 p+p^{2} \sum_{\substack{a=0 \\
a^{3}+b^{3}+1 \equiv 0 \bmod p \\
a+b+1 \equiv 0 \bmod p}}^{p-1} \sum_{\substack{a=0 \\
a+1}}^{p-1} \sum_{\substack{a=0 \\
a^{3}+b^{3}+1 \equiv 0 \bmod p}}^{p-1} \sum_{b-1}^{p-1} 1 .
\end{aligned}
$$

It is clear that the conditions $a^{3}+b^{3}+1 \equiv 0 \bmod p$ and $a+b+1 \equiv 0 \bmod p(0 \leq a, b \leq p-1)$ imply $a(a+1) \equiv 0 \bmod p$ and $a+b+1 \equiv 0 \bmod p$, or $(a, b)=(0, p-1)$ and $(a, b)=(p-1,0)$. So we have

$$
p^{2} \sum_{\substack{a=0 \\ a^{3}+b^{3}+1 \equiv 0 \bmod p \\ a+b+1 \equiv 0 \bmod p}}^{p-1} \sum_{\substack{b=0 \\ a-1}}^{p-2} 1=2 p^{2} .
$$

From (3), (7), Lemma 2, and the properties of Gauss sums we have

$$
\begin{aligned}
p \sum_{\substack{a=0 \\
a^{3}+b^{3}+1 \equiv 0 \bmod p}}^{p-1} \sum_{b=0}^{p-1} & =\sum_{m=0}^{p-1} \sum_{a=0}^{p-1} \sum_{b=0}^{p-1} e\left(\frac{m\left(a^{3}+b^{3}+1\right)}{p}\right) \\
& =p^{2}+\sum_{m=1}^{p-1} e\left(\frac{m}{p}\right)\left(\sum_{a=0}^{p-1} e\left(\frac{m a^{3}}{p}\right)\right)^{2} \\
& =p^{2}+\sum_{m=1}^{p-1} e\left(\frac{m}{p}\right)(\bar{\psi}(m) \tau(\psi)+\psi(m) \tau(\bar{\psi}))^{2} \\
& =p^{2}+\sum_{m=1}^{p-1} e\left(\frac{m}{p}\right)\left(\psi(m) \tau^{2}(\psi)+2 p+\bar{\psi}(m) \tau^{2}(\bar{\psi})\right) \\
& =p^{2}+\tau^{3}(\psi)-2 p+\tau^{3}(\bar{\psi})=p^{2}-2 p+d p .
\end{aligned}
$$

Combining (9), (10), and (11), we have the identity

$$
\sum_{m=1}^{p-1}\left(\sum_{a=0}^{p-1} e\left(\frac{m a^{3}+a}{p}\right)\right)^{3}=2 p^{2}-p d
$$

This proves Lemma 3.

\section{Proofs of the theorems}

We achieve our main results in this part. First, we prove Theorem 1 . For any integer $m$ with $(m, p)=1$, from $(7)$ and Lemma 2 we have

$$
\begin{aligned}
A^{3}(3 m) & =\left(\sum_{a=0}^{p-1} e\left(\frac{3 m a^{3}}{p}\right)\right)^{3}=(\bar{\psi}(3 m) \tau(\psi)+\psi(3 m) \tau(\bar{\psi}))^{3} \\
& =\tau^{3}(\psi)+\tau^{3}(\bar{\psi})+3 p(\bar{\psi}(3 m) \tau(\psi)+\psi(3 m) \tau(\bar{\psi}))=d p+3 p A(3 m) .
\end{aligned}
$$


Applying (7) and Lemmas 1 and 2, we have

$$
\begin{aligned}
& \sum_{m=1}^{p-1} A(3 m)\left(\sum_{a=0}^{p-1} e\left(\frac{m a^{3}+a}{p}\right)\right)^{3} \\
& \quad=\sum_{m=1}^{p-1}(\bar{\psi}(3 m) \tau(\psi)+\psi(3 m) \tau(\bar{\psi}))\left(\sum_{a=0}^{p-1} e\left(\frac{m a^{3}+a}{p}\right)\right)^{3} \\
& \quad=\bar{\psi}(3) \tau(\psi)\left(\psi(3) p \tau^{2}(\psi)-3 p \tau(\bar{\psi})\right)+\psi(3) \tau(\bar{\psi})\left(\bar{\psi}(3) p \tau^{2}(\bar{\psi})-3 p \tau(\psi)\right) \\
& \quad=p\left(\tau^{3}(\psi)+\tau^{3}(\bar{\psi})\right)-3 p^{2}(\psi(3)+\bar{\psi}(3))=d p^{2}+3 p^{2}-3 p^{2}(1+\psi(3)+\bar{\psi}(3)) \\
& \quad=p^{2}(d+3),
\end{aligned}
$$

where we have used the identity $1+\psi(3)+\bar{\psi}(3)=0$.

Applying Lemmas 1, 2, and 3 and (7), we have

$$
\begin{aligned}
\sum_{m=1}^{p-1}\left(\sum_{a=0}^{p-1} e\left(\frac{m a^{3}}{p}\right)\right)^{2}\left(\sum_{a=0}^{p-1} e\left(\frac{m a^{3}+a}{p}\right)\right)^{3} \\
=\sum_{m=1}^{p-1}(\bar{\psi}(m) \tau(\psi)+\psi(m) \tau(\bar{\psi}))^{2}\left(\sum_{a=0}^{p-1} e\left(\frac{m a^{3}+a}{p}\right)\right)^{3} \\
=2 p\left(2 p^{2}-d p\right)+\tau^{2}(\psi)\left(\bar{\psi}(3) p \tau^{2}(\bar{\psi})-3 p \tau(\psi)\right) \\
\quad+\tau^{2}(\bar{\psi})\left(\psi(3) p \tau^{2}(\psi)-3 p \tau(\bar{\psi})\right) \\
=2 p^{2}(2 p-d)+(\psi(3)+\bar{\psi}(3)) p^{3}-3 p\left(\tau^{3}(\psi)+\tau^{3}(\bar{\psi})\right) \\
=p^{2}(3 p-5 d) .
\end{aligned}
$$

Applying Lemmas 1, 2, and 3 and (12), we have

$$
\begin{aligned}
& \sum_{m=1}^{p-1}\left(\sum_{a=0}^{p-1} e\left(\frac{3 m a^{3}}{p}\right)\right)^{3}\left(\sum_{a=0}^{p-1} e\left(\frac{m a^{3}+a}{p}\right)\right)^{3} \\
& \quad=\sum_{m=1}^{p-1}(\bar{\psi}(3 m) \tau(\psi)+\psi(3 m) \tau(\bar{\psi}))^{3}\left(\sum_{a=0}^{p-1} e\left(\frac{m a^{3}+a}{p}\right)\right)^{3} \\
& =d p \sum_{m=1}^{p-1}\left(\sum_{a=0}^{p-1} e\left(\frac{m a^{3}+a}{p}\right)\right)^{3}+3 p \sum_{m=1}^{p-1} A(3 m)\left(\sum_{a=0}^{p-1} e\left(\frac{m a^{3}+a}{p}\right)\right)^{3} \\
& \quad=d p\left(2 p^{2}-p d\right)+3 p\left(3 p^{2}+d p^{2}\right)=p^{2}\left(5 d p+9 p-d^{2}\right) .
\end{aligned}
$$

Now Theorem 1 follows from (13), (14), and (15).

If $p \equiv 1 \bmod 3$ and 3 is a cubic residue $\bmod p$, then $\psi(3)=\bar{\psi}(3)=1$. From Lemma 3 we have

$$
H_{0}(1, p)=2 p^{2}-p d
$$


From (7) and Lemmas 1 and 2 we have

$$
\begin{aligned}
H_{1}(1, p) & =\tau(\psi)\left(p \tau^{2}(\psi)-3 p \tau(\bar{\psi})\right)+\tau(\bar{\psi})\left(p \tau^{2}(\bar{\psi})-3 p \tau(\psi)\right) \\
& =p\left(\tau^{3}(\psi)+\tau^{3}(\bar{\psi})\right)-6 p^{2}=d p^{2}-6 p^{2} .
\end{aligned}
$$

From (7) and Lemmas 1, 2, and 3 we also have

$$
\begin{aligned}
H_{2}(1, p) & =2 p H_{0}(1, p)+\tau^{2}(\bar{\psi})\left(p \tau^{2}(\psi)-3 p \tau(\bar{\psi})\right)+\tau^{2}(\psi)\left(p \tau^{2}(\bar{\psi})-3 p \tau(\psi)\right) \\
& =2 p^{2}(2 p-d)+2 p^{3}-3 p\left(\tau^{3}(\psi)+\tau^{3}(\bar{\psi})\right)=p^{2}(6 p-5 d) .
\end{aligned}
$$

If $k \geq 3$, then applying (12), we have

$$
\begin{aligned}
H_{k}(1, p) & =\sum_{m=1}^{p-1} A^{k}(m)\left(\sum_{a=0}^{p-1} e\left(\frac{m a^{3}+a}{p}\right)\right)^{3} \\
& =\sum_{m=1}^{p-1} A^{k-3}(m)(d p+3 p A(m))\left(\sum_{a=0}^{p-1} e\left(\frac{m a^{3}+a}{p}\right)\right)^{3} \\
& =3 p H_{k-2}(1, p)+d p H_{k-3}(1, p) .
\end{aligned}
$$

Now Theorem 2 follows from (16), (17), (18), and (19).

This completes the proofs of all our results.

\section{Conclusion}

The main work of this paper includes two theorems. In Theorem 1, we obtained some exact values of (2) when $k=1,2$, and 3 . In Theorem 2 , we showed that $H_{k}(1, p)$ satisfies an interesting third-order linear recurrence formula. These works not only profoundly reveal the regularity of a certain hybrid power mean of the trigonometric sums, but also provide some new ideas and methods for further study of such problems.

\section{Acknowledgements}

The authors would like to thank the Editor and referees for their very helpful and detailed comments, which have significantly improved the presentation of this paper.

\section{Funding}

This work is supported by the N.S.F. (11771351 and 11826205) of P.R. China.

Availability of data and materials

Data sharing not applicable to this article as no datasets were generated or analyzed during the current study.

Competing interests

The authors declare that there are no conflicts of interest regarding the publication of this paper.

Authors' contributions

Both authors have equally contributed to this work. Both authors read and approved the final manuscript.

\section{Publisher's Note}

Springer Nature remains neutral with regard to jurisdictional claims in published maps and institutional affiliations. Received: 26 March 2020 Accepted: 27 April 2020 Published online: 15 May 2020 
References

1. Chen, L., Hu, J.Y.: A linear recurrence formula involving cubic Gauss sums and Kloosterman sums. Acta Math. Sinica (Chin. Ser.) 61, 67-72 (2018)

2. Li, X.X., Hu, J.Y.: The hybrid power mean quartic Gauss sums and Kloosterman sums. Open Math. 15, 151-156 (2017)

3. Zhang, H., Zhang, W.P.: The fourth power mean of two-term exponential sums and its application. Math. Rep. 19, 75-83 (2017)

4. Zhang, W.P., Hu, J.Y.: The number of solutions of the diagonal cubic congruence equation mod p. Math. Rep. 20, 73-80 (2018)

5. Chen, Z.Y., Zhang, W.P.: On the fourth-order linear recurrence formula related to classical Gauss sums. Open Math. 15, $1251-1255(2017)$

6. Zhang, W.P., Liu, H.N.: On the general Gauss sums and their fourth power mean. Osaka J. Math. 42, 189-199 (2005)

7. Han, D.: A hybrid mean value involving two-term exponential sums and polynomial character sums. Czechoslov. Math. J. 64, 53-62 (2014)

8. Zhang, W.P., Han, D.: On the sixth power mean of the two-term exponential sums. J. Number Theory 136, 403-413 (2014)

9. Berndt, B.C., Evans, R.J.: The determination of Gauss sums. Bull. Am. Math. Soc. 5, 107-128 (1981)

10. Berndt, B.C., Evans, R.J.: Sums of Gauss, Jacobi, and Jacobsthal. J. Number Theory 11, 349-389 (1979)

11. Kim, T., Kim, D.S.: Degenerate polyexponential functions and degenerate Bell polynomials. J. Math. Anal. Appl. 487(2), $124017(2020)$

12. Kim, H.S., Kim, T.: On certain values of p-adic q-L-functions. Rep. Fac. Sci. Eng. Saga Univ., Math. 23(1-2), 1-7 (1995)

13. Apostol, T.M.: Introduction to Analytic Number Theory. Springer, New York (1976)

14. Hua, L.K.: Introduction to Number Theory. Science Press, Beijing (1979)

\section{Submit your manuscript to a SpringerOpen ${ }^{\circ}$ journal and benefit from:}

- Convenient online submission

- Rigorous peer review

- Open access: articles freely available online

- High visibility within the field

- Retaining the copyright to your article

Submit your next manuscript at springeropen.com 